Vol. 9(4), pp. 239-247, 28 J a nuary, 2015

DOI: 10.5897/AJ MR2014.7289

Artic le Number: C20B2A250422

ISSN 1996-0808

Copyright (C) 2015

African Journal of Microbiology Research

Author(s) reta in the copyright of this a ricle

http://www.a cademic joumals.org/AJ MR

\title{
Molecular characterization and phenotypic analysis of multidrug-resistant Escherichia coli and Klebsiella pneumoniae isolates from a tertiary-care hospital in Yunnan Province, China
}

\author{
Tan, H. L. ${ }^{1 \#}$, Wang, Y. ${ }^{2,3 \#}$, Cheng, X. Q. ${ }^{2 \#}$, Huang, Y. M. ${ }^{1}$ and Zhang, L. J. ${ }^{2 *}$ \\ ${ }^{1}$ Department of Clinical Microbiology Laboratory, The Third People's Hospital of Yunnan Province, Kunming , People's \\ Republic of China. \\ ${ }^{2}$ The National Institute for Communicable Disease Control and Prevention, China CDC, Beijing, People's Republic of \\ China. \\ ${ }^{3}$ College of Animal Science and Technology, Shihezi University, Shihezi, Xinjiang Province, People's Republic of China.
}

Received 20 November, 2011; Accepted 19 January, 2015

\begin{abstract}
Due to the increasing number of multidrug-resistant (MDR) isolates of Escherichia coli and Klebsiella pneumoniae reported from the Third People's Hospital of Yunnan Province, an investigation was conducted to better understand the phenotype and molecular characterization of the local isolates. Twenty three non-duplicate $E$. coli isolates and nine $K$. pneumoniae isolates were recovered from hospitalized patients and identified and tested for antimicrobial susceptibility using the VITEKw2 system. Drug-resistant genes were amplified and sequenced, and pulsed-field gel electrophoresis (PFGE) and multilocus sequence typing (MLST) analyses were performed on the tested isolates. All of the isolates, except for one extensively drug-resistant (XDR) $K$. pneumoniae isolate, were demonstrated to be MDR, and $100 \%$ of the $E$. coli and $K$. pneumoniae were resistant to ampicillin, cefuroxime, cefazolin and ceftriaxone. Of the isolates, $69.6 \%$ of the $E$. coli and $100 \%$ of the $K$. pneumoniae isolates were bla $\mathrm{C}_{\mathrm{CTX}-\mathrm{M}}$ positive, with CTX-M-55 and CTX-M-15 as the leading genotypes. All $K$. pneumoniae isolates shared bla $\mathrm{SHV}_{\mathrm{SH}}$ genes with the dominant SHV-11 genotype. A total of $87.5 \% \mathrm{E}$. coli and $77.8 \%$ of $K$. pneumoniae carried the ISEcp1, $91.3 \%$ of $E$. coli and $77.8 \%$ of $K$. pneumoniae shared the int1 gene, and $44.4 \%$ of $K$. pneumoniae presented the ISCR1 gene. A large genetic heterogeneity of $K$. pneumoniae and E. coli isolates was confirmed by MLST and PFGE analyses. The high frequency of MDR E. coli and $K$. pneumoniae in local areas may be a substantial challenge for infection control.
\end{abstract}

Key words: Klebsiella pneumonia, Escherichia coli, molecular characterization, multidrug-resistant.

\section{INTRODUCTION}

Multidrug-resistant gram-negative bacteria (GNB) have increased globally in recent years (Adams-Sapper et al., 2012; Drees et al., 2014; Tacconelli et al., 2014). These drug-resistant GNB can not only cause outbreaks in community settings (Smith et al., 2008; Wei et al., 2005) but also spread throughout the world in many ways, including by person-to-person transmission following foreign travel and through widely distributed food 
products contaminated with drug-resistant GNB (Peirano and Pitout, 2010; Kumarasamy et al., 2010; Johnson et al., 2010). Clinical settings are important reservoirs and sources of drug-resistant GNB pathogens and drugresistance genes. Antimicrobial agents used in hospitals may facilitate the transfer of mobile drug-resistance genes across different lineages of the same bacterial species or across different bacterial species by horizontal gene transfer. Notably, the emergence and spread of MDR E. coli and $K$. pneumoniae are associated with significant morbidity and mortality (Sievert et al., 2013; Carbonne et al., 2013). In recent years, a significant increase in the number of MDR isolates of $E$. coli and $K$. pneumoniae has been reported from the clinical microbiology laboratory in the Third People's Hospital of Yunnan Province (Tan et al., 2014), a tertiary-care hospital in Kunming, the capital city of Yunnan Province, China. To better understand the phenotype and molecular characterization of the drug resistance determinants of local E. coli and K. pneumoniae isolates and to monitor the emergence of novel antimicrobial resistance isolates, a joint investigation was conducted between the National Institute for Communicable Disease Control and Prevention, China CDC, People's Republic of China and the Third People's Hospital of Yunnan Province, People's Republic of China, from June to September 2013.

\section{MATERIALS AND METHODS}

\section{Ethics statement}

All protocols in the study were approved by the institutional ethics committee of the Third People's Hospital of Yunnan Province. Written consent was given by the patients for the use of pathogens isolated from them and for the evaluation of information in their medical records for research purposes. All samples and information were made anonymous.

\section{Study design, specimen collection and patient demographics}

This study was a prospective investigation of $E$. coli and $K$. pneumoniae isolates recovered from patients in the microbiology laboratories of the Third People's Hospital of Yunnan Province (a 1,000-bed tertiary-care hospital serving approximately 110,000 inpatients per year) between June and September 2013. Among these patients who had positive $E$. coli cultures, four were from the department of nephrology, three were from the intensive care unit (ICU), three were from the department of urinary surgery, three were from the department of geriatrics, two were from the department of traditional chinese medicine, one was from the department of physiatry, one was from the department of endocrinology, one was from the department of gastroenterology, one was from the department of general surgery, one was from the department of respiration, one was from the department of internal neurology, one was from the department of neurosurgery and one was from the department of chest surgery. Of the patients who had positive $K$. pneumoniae cultures, three were from the ICU, two were from the department of respiration, one was from the department of physiatry, one was from the department of neurosurgery, one was from the department of nephrology and one was from the department of urinary surgery.

All patient demographics were recorded, including age, sex, hospital ward, and the types of specimens. The first isolate was chosen in the case of duplicate patient samples.

\section{Identification and antimicrobial susceptibility testing of isolates}

Bacteria were isolated from patients according to the standard protocol from the Manual of Clinical Microbiology Laboratory (Zhou et al., 2010). Identification and antimicrobial susceptibility testing of isolates were conducted with standard biochemical tests using the bioMérieux VITEK-2 system following the manufacturer's instructions. Identification of isolates to the species level was further confirmed by amplifying and sequencing the 16S rRNA gene with universal prokaryotic bacterial 16S rRNA primers (Weisburg et al., 1991). A total of 21 drugs were included in the antimicrobial susceptibility testing: ampicillin (AMP), piperacillin/tazobactam (TZP), ampicillin/sulbactam (SAM), cefuroxime (CXM), cefazolin (CFZ), ceftriaxone (CRO), ceftazidime (CAZ), cefoperazone (SCF), cefepime (FEP), cefotetan (CTT), ertapenem (ETP), meropenem (MEM), imipenem (IMP), aztreonam (ATM), ciprofloxacin (CIP), levofloxacin (LEV), gentamicin (GM), tobramycin (TM), kanamycin (AN), trimethoprim-sulfamethoxazole (SXT) and furadantin (FD). The E. coli strains: ATCC 25922 and ATCC 35218 and the $K$. pneumoniae strain ATCC 700603 were used as quality control strains. According to the standardized international definitions of multidrug-resistant (MDR) (Magiorakos et al., 2012), MDR was defined as resistant to at least one agent in three or more agent categories, extensively drug-resistant (XDR) was defined as resistant to at least one agent in all but two or fewer antimicrobial categories, and pandrug-resistant (PDR) was defined as resistant to all agents in all antimicrobial categories.

\section{Amplification and sequencing of the blagene and gene- capturing elements}

Genomic DNA was extracted using a DNeasy® Blood and Tissue Kit (QIAGEN, Hilden, German, Cat No. 69506) and plasmid DNA was extracted using a high-purification plasmid mini-preparation kit (BioTeke Corporation, Beijing, China, Cat\#DP1002). PCR was performed using a SensoQuest LabCycler standard plus (SensoQuest $\mathrm{GmbH}$, Goettingen, Germany) with Taq DNA polymerase (SBS Genetech Co., Ltd, China, Lot\#042512). All bacteria were subjected to PCR for the detection of the bla genes with the primers listed in Table 1 using genomic and plasmid DNA of the bacteria as templates. The PCR conditions were based on the reference sources listed in Table 1 . The PCR products were sequenced in both directions by two separate commercial sequencing companies in China (Beijing Tsingke BioTech Co., Ltd.

*Corresponding author. E-mail: zhanglijuan@icdc.cn. Tel/Fax: 0086-10-58900780.

${ }^{\#}$ Contributed equally to this work.

Author(s) agree that this article remain permanently open access under the terms of the Creative Commons Attribution License 4.0International License 
Table 1. Primers used for PCR amplification of the bla gene and gene-capturing elements

\begin{tabular}{|c|c|c|c|c|}
\hline Target & Primer name & Primer sequence (5'-3') & $\begin{array}{l}\text { Product } \\
\text { size (bp) }\end{array}$ & Reference \\
\hline \multicolumn{5}{|l|}{ bla gene } \\
\hline \multirow[t]{2}{*}{ TEM } & TEM-F & TCCGCTCATGAGACAATAACC & 931 & Sturenburg et al., 2004 \\
\hline & TEM-R & TTGGTCTGACAGTTACCAATGC & & \\
\hline \multirow[t]{2}{*}{ SHV } & SHV-F & TGGTTATGCGTTATATTCGCC & 868 & Pai et al.,1999 \\
\hline & SHV-R & GGTTAGCGTTGCCAGTGCT & & \\
\hline \multirow[t]{2}{*}{ СТХ-M } & CTX-F & TCTTCCAGAATAAGGAATCCC & 909 & Sturenburg et al., 2004 \\
\hline & CTX-R & CCGTTTCCGCTATTACAAAC & & \\
\hline \multirow[t]{2}{*}{ VEB } & VEB-F1 & GATAGGAGTACAGACATATG & 914 & Pastera'n et al., 2006 \\
\hline & VEB-R1 & TTTATTCAAATAGTAATTCCACG & & \\
\hline \multirow[t]{2}{*}{ OXA-2 group } & OXA-2-F & AAGAAACGCTACTCGCCTGC & 478 & Yan et al., 2006 \\
\hline & OXA-2-R & ССАСТСААСССАТССТАССС & & \\
\hline \multirow[t]{2}{*}{ OXA-10 group } & OXA-10-F & GTCTTTCGAGTACGGCATTA & 720 & Bert et al., 2002 \\
\hline & OXA-10-R & ATTTTCTTAGCGGCAACTTAC & & \\
\hline \multirow[t]{2}{*}{ PER } & PER-F & ATGAATGTCATCACAAAATG & 927 & Celenza et al., 2006 \\
\hline & PER-R & TCAATCCGGACTCACT & & \\
\hline \multirow[t]{2}{*}{ GES } & GES-F & ATGCGCTTCATTCACGCAC & 864 & Vourli et al., 2004 \\
\hline & GES-R & CTATTTGTCCGTGCTCAGG & & \\
\hline \multicolumn{5}{|c|}{ Gene-capturing elements } \\
\hline \multirow[t]{2}{*}{ int1 } & int $1 \mathrm{~F}$ & CCTCCCGCACGATGATC & 280 & Chen et al., 2013 \\
\hline & int $1 \mathrm{R}$ & TCCACGCATCGTCAGGC & & \\
\hline \multirow[t]{2}{*}{ ISCR1 } & CR1 F & ATGTCTCTGGCAAGGAACGC & 1450 & Chen et al., 2013 \\
\hline & CR1 R & AGACGACTCTGTGATGGATC & & \\
\hline \multirow[t]{2}{*}{ ISEcp1 } & IS-F & GTGCCCAAGGGGAGTGTATG & 615 & Kiratisin et al., 2007 \\
\hline & IS-R & ACYTTACTGGTRCTGCACAT & & \\
\hline
\end{tabular}

and Shanghai Sangon BioTech Co., Ltd.). Sequencing was performed with an ABI 3100 genetic analyzer (Applied Biosystems) using the BigDye Terminator v3.1 cycle sequencing kit (Applied Biosystems, Foster City, CA) according to the manufacturer's instructions. The sequences were analyzed using the nucleotide BLAST program (http://blast.ncbi.nlm.nih.gov/).

ISEcp1, which plays a key role in gene transfer (Kiiru et al., 2013; Dhanji et al., 2011; Tian et al., 2011), is often located in the upstream region preceding bla $\mathrm{C}_{\mathrm{CTX}-\mathrm{M}}$, an emerging and highly prevalent CTX-M genotype among $E$. coli and $K$. pneumoniae strains worldwide. The ISEcp1 distribution among the isolates was assayed by PCR using primers targeting ISEcp1 (Table 1).

In addition, the presence of the key gene-capturing elements int1 and ISCR1 was determined by PCR using primers targeting int1 and ISCR1 (Table 1) and genomic DNA (gDNA) from the bacteria because the expression of int1 and ISCR1 is closely associated with the dissemination of MDR bacteria among clinical isolates.

\section{MLST and PFGE analysis}

To study the clonal relationships of the isolates tested, MLST and PFGE were conducted. According to the MLST protocol described online (www.pasteur.fr/mlst), 7 genes, including gapA, infB, $m d h$, pgi, phoE, rpoB and ton $B$, were used for genotyping the $K$. pneumoniae isolates, and 8 genes, including $\operatorname{din} B$, icd $A, p a b B$, polB, putP, $\operatorname{trp} A, \operatorname{trp} B$ and uidA, were used for genotyping the $E$. coli isolates. The PCR products from MLST were sequenced as described in the aforementioned methods. Sequence types (STs) were assigned using the MLST database (www.pasteur.fr/mlst).
PFGE analysis of E. coli was performed using a CHEF Mapper XA apparatus (Bio-Rad Laboratories, Hercules, CA) according to the standard protocol of the International Molecular Subtyping Network for Foodborne Disease Surveillance (http://www.pulsenetinternational.org/protocols/), and the PFGE protocol for K. pneumoniae was performed as previously described (Han et al., 2013). The PFGE banding patterns were analyzed using Fingerprinting II Software, version 3.0 (Bio-Rad Laboratories, Hercules, CA). A PFGE group was defined as having more than $80 \%$ similarity.

\section{RESULTS}

\section{Specimen collection and patient demographics}

During the study period, a total of 23 non-duplicate E. coli isolates and nine $K$. pneumoniae isolates were obtained from hospitalized patients. The proportions of males and females were $61.0 \% \quad(n=19)$ and $41.2 \% \quad(n=13)$, respectively, and the median age of the patients was 61 years (range $3.0-88.0$ years). $23 \mathrm{E}$. coli isolates were recovered from 12 urine specimens, four blood cultures, three sputum specimens, two secretion specimens, one pus specimen and one sterile cavity fluid specimen, while nine $K$. pneumoniae isolates were isolated from five sputum specimens, two urine specimens, one blood culture and one throat swab specimen. 
Table 2. Drug-resistance rates of $E$. coli and K. pneumonia

\begin{tabular}{|c|c|c|c|c|c|c|c|c|}
\hline Antimicrobial category & Agent $^{a}$ & \multicolumn{3}{|c|}{$\begin{array}{c}\text { E. coli \% } \\
\text { (No. positive/No. tested) }\end{array}$} & \multicolumn{3}{|c|}{$\begin{array}{c}\text { K. pneumoniae \% } \\
\text { (No. positive/No. tested) }\end{array}$} & $\mathbf{p}$ \\
\hline Ampicillin & AMP & 0 & 0 & $100(23 / 23)$ & & & $100(9 / 9)$ & \\
\hline penicillin + beta-lactamase inhibitors & SAM & $4.3(1 / 23)$ & $13.0(3 / 23)$ & $82.6(19 / 23)$ & & $11.1(1 / 9)$ & $88.9(8 / 9)$ & $<1.0$ \\
\hline \multirow[t]{2}{*}{1 st and 2nd generation cephalosporin } & CXM & 0 & 0 & $100(23 / 23)$ & 0 & 0 & $100(9 / 9)$ & \\
\hline & CFZ & 0 & 0 & $100(23 / 23)$ & 0 & 0 & $100 \%(9 / 9)$ & \\
\hline \multirow{3}{*}{$3^{\text {rd }}$ and $4^{\text {th }}$ generation cephalosporin } & CAZ & $47.8(11 / 23)$ & 0 & $52.2(12 / 23)$ & $11.1(1 / 9)$ & $11.1(1 / 9)$ & $77.8(7 / 9)$ & 0.2 \\
\hline & SCF & $73.9(17 / 23)$ & $17.4(4 / 23)$ & $2 / 23$ & $44.4(4 / 9)$ & $33.3(3 / 9)$ & $22.2(2 / 9)$ & 0.6 \\
\hline & FEP & $52.2(12 / 23)$ & $4.3(1 / 23)$ & $43.5(10 / 23)$ & $33.3(3 / 9)$ & 11.1(1/9) & $55.6(5 / 9)$ & 0.7 \\
\hline Cephamycin & CTT & $91.3(21 / 23)$ & $4.3(1 / 23)$ & $4.3(1 / 23)$ & $77.8(7 / 9)$ & 11.1(1/9) & 11.1(1/9) & 0.5 \\
\hline \multirow[t]{2}{*}{ Carbapenem } & ETP & $100(23 / 23)$ & 0 & 0 & $88.9(8 / 9)$ & 0 & 11.1(1/9) & \\
\hline & MEM & $100(23 / 23)$ & 0 & 0 & $88.9(7 / 9)$ & 0 & 11.1(1/9) & \\
\hline \multirow[t]{3}{*}{ Aminoglycoside } & GM & $34.8(8 / 23)$ & 0 & $65.2(15 / 23)$ & $55.6(5 / 9)$ & 0 & $44.4(4 / 9)$ & 0.4 \\
\hline & TM & $39.1(9 / 23)$ & $21.7(5 / 23)$ & $39.1(9 / 23)$ & $33.3(3 / 9)$ & $11.1(1 / 9)$ & $55.6(5 / 9)$ & 0.5 \\
\hline & AN & $95.6(22 / 23)$ & $4.3(1 / 23)$ & 0 & $55.6(5 / 9)$ & 0 & $44.4(4 / 9)$ & 0.004 \\
\hline Folate pathway inhibitors & SXT & $39.1(9 / 23)$ & 0 & $60.9(14 / 23)$ & $44.4(4 / 9)$ & 0 & $55.6(5 / 9)$ & $<1.0$ \\
\hline Nitrofurantoin & FD & $65.2(15 / 23)$ & $30.4(7 / 23)$ & $4.3(1 / 23)$ & $22.2(2 / 9)$ & $11.1 \%(1 / 9)$ & $66.7 \%(6 / 9)$ & 0.0006 \\
\hline Total & & $57.0(223 / 391)$ & $12.5(23 / 184)$ & $63.8(235 / 368)$ & $49.3(71 / 144)$ & $19.4(14 / 72)$ & $54.5(103 / 189)$ & $<0.0001$ \\
\hline
\end{tabular}

${ }^{a}$ Abbreviations of drugs: AMP, Ampicillin; TZP, Piperacillin/Tazobactam; SAM, Ampicillin/Sulbactam; CXM, Cefuroxime; CFZ, Cefazolin; CRO, Ceftriaxone; CAZ, Ceftazidime; SCF, Cefoperazone; FEP, Cefepime; CTT, Cefotetan; ETP, Ertapenem; MEM, Meropenem; IMP, Imipenem; ATM, Aztreonam; CIP, Ciprofloxacin; LEV, Levofloxacin; GM, Gentamycin; TM,

Tobramycin; AN, Kanamycin, SXT, Trimethoprim-Sulfamethoxazole; FD, Furadantin. ${ }^{\text {bS }}$, susceptible; I, intermediate; R, resistant.

\section{Antimicrobial susceptibility}

Detailed information on the resistance rates to all tested drugs is listed in Table 2. All of the E. coli and $K$. pneumoniae isolates exhibited resistance to AMP, CXM and CFZ and even to CRO (3rd generation cephalosporin). In addition, most $K$. pneumoniae and $E$. coli isolates were resistant to ATM $(88.9 \%$ for $K$. pneumoniae and $87.0 \%$ for $E$. coli), SAM ( $88.9 \%$ for $K$. pneumoniae and $82.6 \%$ for E. coli) and CAZ $(77.8 \%$ for $K$. pneumoniae and $52.2 \%$ for E. coli). Notably, higher resistance rates to CIP, LEV, GM, TM, AN, SXT and FD were detected in $55.6 \%, 33.3 \%, 44.4 \%, 55.6 \%$, $44.4 \%, 55.6 \%$ and $66.7 \%$ of $K$. pneumoniae. 
Table 3. Molecular characterization of blagenes among $E$. coli and $K$. pneumoniae isolates

\begin{tabular}{llc}
\hline Organism & Genotype of bla gene & No. of isolates \\
\hline \multirow{5}{*}{ E. coli } & TEM-1 & 1 \\
& TEM-1, CTX-M-55 & 5 \\
& TEM-1, CTX-M-15 & 2 \\
& TEM-1, CTX-M-3 & 1 \\
& CTX-M-15 & 5 \\
& CTX-M-55 & 3 \\
& TEM-1, CTX-M-55, SHV-11 & 1 \\
& TEM-1, CTX-M-15, SHV-1/148 & 1 \\
& TEM-1, CTX-M-55, SHV-2 & 1 \\
& TEM-1, CTX-M-15,SHV-1b-b & 1 \\
& CTX-M-55, SHV-11 & 3 \\
& CTX-M-15, SHV-108 & 1 \\
& CTX-M-15, SHV-11 & 1 \\
\hline
\end{tabular}

Table 4. Sequence analysis of the ISEcp1-carrying bla

\begin{tabular}{lllc}
\hline Organism & Type of bla CTX-M $_{\text {CIS element }}$ & No. of isolates \\
\hline \multirow{4}{*}{ E. coli } & CTX-M-3 & IS26 & 1 \\
& CTX-M-55 & ISEcp1 & 6 \\
& CTX-M-15 & ISEcp1 & 4 \\
\multirow{5}{*}{ K. pneumoniae } & CTX-M-15 & IS1 interrupting ISEcp1 & 2 \\
& CTX-M-15 & IS1 & 1 \\
& CTX-M-55 & IS1 interrupting ISEcp1 & 4 \\
& CTX-M-15 & ISEcp1 & 3 \\
\hline
\end{tabular}

The isolates, and higher resistance to CIP, LEV, GM, TM and SXT was seen in $91.3 \%, 82.6 \%, 65.2 \%, 39.1 \%$, and $60.9 \%$ of $\mathrm{E}$. coli isolates, respectively. The lowest resistance rates were observed for carbapenems, and all $23 \mathrm{E}$. coli isolates and eight of nine $K$. pneumoniae isolates, except for one XDR isolate, were sensitive to ETP, MEM and IMP (Table 2). In this study, 100\% (23/23) of $E$. coli and $88.9 \%(8 / 9)$ of $K$. pneumoniae isolates (except one isolate defined as XDR) were confirmed as being MDR according to the standardized international definitions for drug resistance.

\section{Amplification and sequencing of the bla genes and gene-capturing elements}

The bla TEM $_{\text {T }}$ and bla ${ }_{\text {CTX }}$ groups were detected in $39.1 \%$ $(9 / 23)$ and $69.6 \%(16 / 23)$ of $E$. coli and $44.4 \%(4 / 9)$ and $100 \%(9 / 9)$ of $K$. pneumoniae, respectively. All of the bla ${ }_{\mathrm{TEM}}$-positive isolates, including the $9 \mathrm{E}$. coli isolates and the $4 \mathrm{~K}$. pneumoniae isolates, encoded bla $\mathrm{TEM}-1_{1}$. For the CTX-M-producing isolates, bla 3, CTX-M-15, and CTX-M-55 was found in 6.2, 43.8 and $50.0 \%$ of $E$. coli isolates and $0,44.4$ and $55.6 \%$ of $K$. pneumoniae isolates, respectively (Table 3 ). All $K$. pneumoniae isolates, but none of the $E$. coli isolates, included bla $a_{\mathrm{SHV}}$ genes, and bla $a_{\mathrm{SHV}}$ genes encoding SHV2, SHV-11, SHV-1b-b, SHV-108, and SHV-148/-1 was identified in $11.1,55.5,11.1,11.1$ and $11.1 \%$ of $K$. pneumoniae, respectively (Table 3 ). Other bla genes, including bla $\mathrm{OXA}_{2}$ group, bla $\mathrm{OXA}_{10}$ group, bla $\mathrm{VEB}_{\mathrm{B}}$, bla $a_{\mathrm{PER}}$ and bla $a_{\mathrm{GES}}$, were not detected in any of the isolates in the study. ISEcP1 was identified in the upstream region of

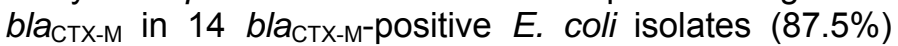

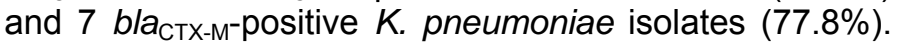
Sequence analysis of the ISEcp1-carrying bla CTX-M $_{\text {is }}$ shown in Table 4. IS1 was found to interrupt the ISEcp1 gene in $2 \mathrm{E}$. coli isolates and $4 \mathrm{~K}$. pneumoniae isolates within the sequences upstream of bla $a_{\mathrm{CTX}-\mathrm{M} \text {. }}$

Amplification and sequencing of int1 and the ISCR1 gene-capturing elements indicated that $91.3 \%(21 / 23)$ of E. coli isolates and $77.8 \%(7 / 9)$ of $K$. pneumoniae isolates carried the int1 gene, and $44.4 \%$ of $K$. pneumoniae and $0 \%$ of $E$. coli contained the ISCR1 gene.

\section{MLST and PFGE typing}

The analysis of MLST sequence types (STs) showed that 
Table 5. MLST profiles of $E$. coli and K. pneumoniae based on bla $\mathrm{C}_{\mathrm{CTX}-\mathrm{M}}$ type

\begin{tabular}{lll}
\hline Organism & blacTX-M type & MLST type (No. of type) \\
\hline & CTX-M-3 & ST7 $(n=1)$ \\
& CTX-M-15 & ST7 $(n=7)$ \\
& & ST7 $(n=3)$ \\
\multirow{3}{*}{ E. coli } & CTX-M-55 & ST6 $(n=3)$ \\
& & ST5 $(n=1)$ \\
& ST2 $(n=1)$ \\
& Non-CTX-M & ST7 $(n=6)$ \\
& ST2 $(n=1)$ \\
K. pneumoniae & CTX-M-15 & ST45S $(n=1) ; \operatorname{ST11}(n=1) ; S T 29(n=1) ; S T 7(n=1)$ \\
& CTX-M-55 & ST395 $(n=3) ; S T 65(n=1) ; S T 629(n=1)$ \\
\hline
\end{tabular}

ST7 was the dominant MLST type among the E. coli isolates, which accounted for $68.8 \%(11 / 16)$ of the bla м-positive E. coli isolates and $85.7 \%(6 / 7)$ of the non-

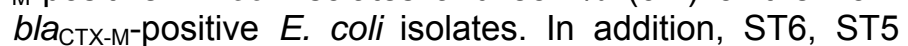
and ST2 were detected in 18.8, 6.3 and $6.3 \%$,

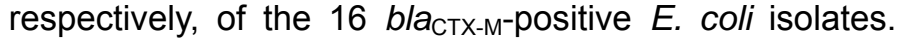
Further, ST6, ST5 and ST2 types uniquely co-existed with CTX-M-55 (Table 5). In contrast, a large diversity of MLST types was identified among the nine bla CTX-M $^{-}$ positive $K$. pneumoniae isolates, and a total of seven types were found. Four CTX-M-15 K. pneumoniae isolates shared different MLST types (Table 5). The five CTX-M-55 K. pneumoniae isolates were divided into three MLST types, in which ST395, ST629 and ST65 accounted for $60 \%(3 / 5), 20 \%(1 / 5)$ and $20 \%(1 / 5)$, respectively.

No banding patterns were obtained by PFGE analysis in three $E$. coli and two $K$. pneumoniae isolates because their DNA samples were consistently auto-digested. In contrast to the MLST typing, a large genetic diversity of $E$. coli isolates was demonstrated by PFGE typing, and 13 clusters were obtained based on the definition of more than $80 \%$ similarity (Figure $1 \mathrm{~A}$ ). However, there was little relatedness among the PFGE types, ST types and CTX$M$ types (Figure 1A). Similarly, the PFGE banding patterns of the seven $K$. pneumoniae isolates were very different (Figure 1B).

\section{DISCUSSION}

The emergence and rapid spread of multidrug-resistant Enterobacteriaceae is a substantial challenge to public health. We first reported the high frequency and molecular characterization of MDR $E$. coli and $K$. pneumoniae in Yunnan Province, China. Although, extended-spectrum beta-lactamases have been recognized among Enterobacteriaceae worldwide (Rossolini et al., 2008; Bonnet, 2004) and are known for their rapid spread in the US, Europe and Asia (Wang et al., 2013; Sidjabat et al., 2009; Johnson et al., 2010), it was remarkable that in this study $100 \%(9 / 9)$ of $K$. pneumoniae and $69.6 \%(16 / 23)$ of $E$. coli carried the

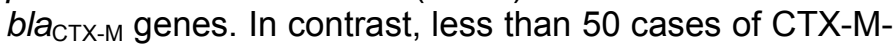
producing $K$. pneumoniae isolates had been described in the United States before 2013 (Wang et al., 2013). Because the isolates recovered from patients were from different hospital departments and displayed high genetic diversity, the high rates of MDR $E$. coli and $K$. pneumoniae isolates in the hospital may be a considerable challenge for antimicrobial drug-use and infection control. Unlike the distributions of the dominant CTX-M genotypes in Beijing (CTX-M-10, 35.9\%) (Li et al., 2012), Hunan Province (CTX-M-14), Jiangxi Province (CTX-M-14), Fujian Province (CTX-M-14) and Guangdong Province (CTX-M-14) (Wang et al., 2012), the CTX-M-55 and CTX-M-15 genotypes were the leading CTX-M genotypes in Yunnan Province in this study and they were identified in 50 and $43.8 \%$ of $E$. coli isolates, $55.6 \%$ and $44.4 \%$ of $K$. pneumoniae isolates, respectively. It is noteworthy that CTX-M-15 is the most prevalent genotype throughout the world (Carbonne et al., 2013; Kiratisin et al., 2007; Wang et al., 2013; Li et al., 2012; Paterson et al., 2003; Parveen et al., 2012), and it has frequently been reported in several parts of China (Li et al., 2012; Wang et al., 2012), however, CTXM-55 has only been described a few times in China. Only one $E$. coli isolate was identified as CTX-M-3 in the study. Strikingly, all of the CTX-M genotypes identified in the study, including CTX-M-15, CTX-M-55 and CTX-M-3, have traditionally been classified in the CTX-M-1 subgroup, and increasing evidence indicates that the CTX-M-1 subgroup of bacteria exhibits higher levels of resistance to several antibiotics than other CTX-M subgroups (Wang et al., 2013; Paterson et al., 2003; Parveen et al., 2012). Here, we propose that the high frequency of CTX-M-1 subgroup isolates may have contributed to the high level of MDR agents identified in the study. A survey conducted from 1998 to 1999 in Thailand, a country neighboring the Yunnan Province, reported the first detection of bla prevalence of bla CTX-M was $52 \%$ (CTX-M-9 only) and 


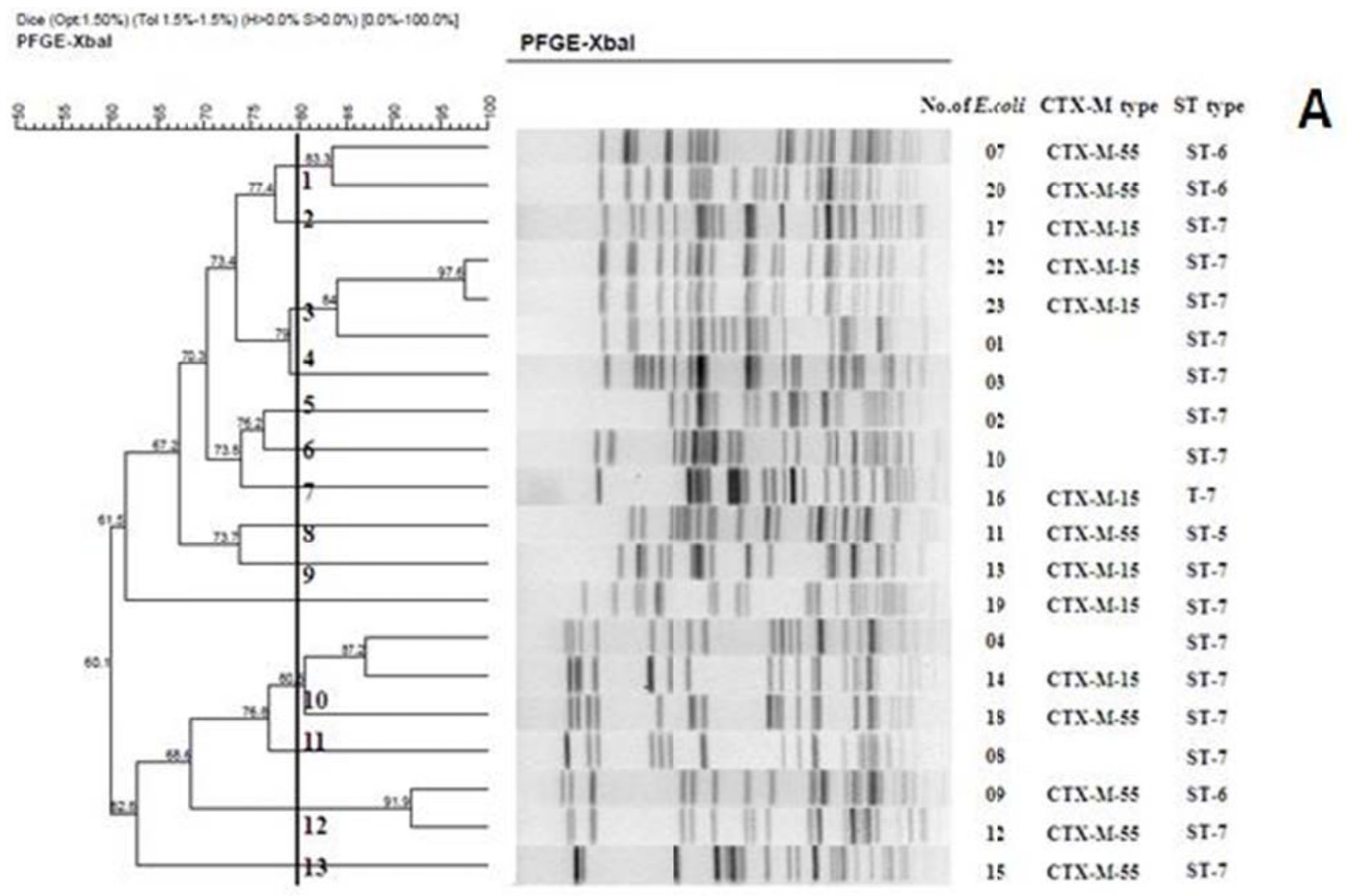

\section{Dice (O.0t 1.50\%) (Tol 1.5\%.1.5\%) (H>0.0\% 500.0\%) $[0.0 \%-100.0 \%]$ \\ PFGE_Xbal_6-36s \\ PFGE_Xbal_6 36s}

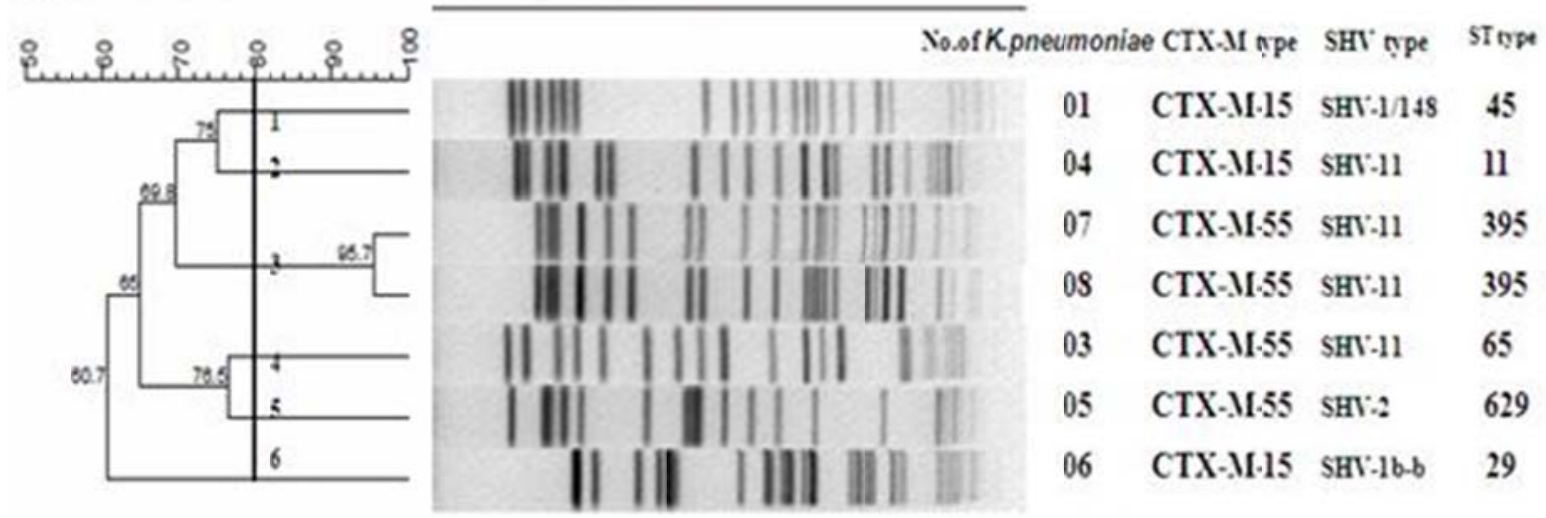

Figure 1. Dendrogram of pulsed-field gel electrophoresis (PFGE) patterns of E. coli (A) and K. pneumoniae (B) isolates and their genetic relatedness to the CTX-M genotype and sequence type (ST), if available.

subsequently increased to $65 \%$ in 2003 (CTX-M-15, 44\%; CTX-M-14, 11\% and CTX-M-9, 10\%) (Chanawong et al., 2007). More recently, the frequency has increased to $99.6 \%$ of bla CTX-M-15, 37.2\%; CTX-M-27, 1.3\%; CTX-M-40, 1.3\% and CTX-M-55, $17.5 \%$ ) and $99.2 \%$ of bla K. pneumoniae (CTX-M-3, 3.2\%; CTX-M-14, 52.4\%; CTX-M-15, 38.9\%; CTX-M-27, $0.8 \%$ and CTX-M-55,
4.7\%) in 2004-2005. These results indicate that CTX-M55 is also an emerging CTX-M genotype in Thailand.

Significantly, an XDR $K$. pneumoniae isolated from the blood culture sample of a 66-year-old male patient exhibited resistance to all the carbapenem drugs tested in the study. This XDR K. pneumoniae exhibited the CTX$M-15$, SHV-1b-b and TEM-1 genotypes, and it also carried the ISEcP1 and ISCR1 gene-capturing elements. 
In this study, TEM-types were less numerous among the $E$. coli and $K$. pneumoniae isolates than CTX-M-types (39.1 vs $69.6 \%$ for $E$. coli and 44.4 vs $100 \%$ for $K$. pneumoniae). This result is not surprising because cefotaxime and ceftriaxone are used nationwide. The $b_{\text {blav }}$ genes were not present in the $E$. coli isolates, but all of the $K$. pneumoniae isolates contained the bla genes. Moreover, these bla $a_{\mathrm{SHV}} K$. pneumoniae isolates all coexisted with CTX-M genes. The dominant SHV genotype was SHV-11, and it was identified in $55.6 \%$ of $K$. pneumoniae isolates, which was significantly higher than that identified in the Beijing area (Li et al., 2012). The other SHV genotypes found in the study includes SHV-2, SHV-1b-b, SHV-108, and SHV-148/-1 was unique, and each accounted for $11.1 \%$ of the isolates.

A high frequency of ISEcp1, which is regarded as having a key role both in drug-resistance gene transfer and as a promoter for bla $a_{\text {CTX-M }}$, was detected in bla $a_{\text {CTX-M }}{ }^{-}$ carrying $E$. coli isolates and $K$. pneumoniae isolates in this study. Two other gene-capturing elements, int 1 and ISCR1, which were previously noted elsewhere (Li et al., 2012) and are involved in multidrug resistance among clinical isolates, were also demonstrated in $E$. coli and $K$. pneumoniae isolates, respectively, further supporting our proposal that these gene-capturing elements might contribute to the high detection rates of MDR E. coli isolates and $K$. pneumoniae isolates in the Yunnan area. In addition, the genetic heterogeneity of $E$. coli and $K$. pneumoniae was noticed among the isolates (Figure 1A and $1 \mathrm{~B}$ ) analyzed in the study, suggesting that the emergence and polyclonal spread of multidrug-resistant $E$. coli and $K$. pneumoniae occurred among clinical isolates with diverse genetic backgrounds.

To summarize, we report for the first time the molecular characterization and phenotypes of $E$. coli and $K$. pneumoniae isolates in Yunnan Province, China. Our data revealed a high frequency of MDR $E$. coli and $K$. pneumoniae isolates among polyclonal strains in local areas. The CTX-M-1 subgroups which are involved in the development of higher levels of drug resistance were highly endemic among hospitalized patients. Active monitoring of novel antibiotic resistance is critical to avoid the rapid spread or outbreaks of these multidrug-resistant isolates in local health care facilities.

\section{REFERENCES}

Adams-Sapper S, Sergeevna-Selezneva J, Tartof S, Raphael E, Diep BA, Perdreau-Remington F, Riley LW (2012). Globally dispersed mobile drug-resistance genes in gram-negative bacterial isolates from patients with bloodstream infections in a US urban general hospital. J. Med. Microbiol. 61:968-74.

Bert F, Branger C, Lambert-Zechovsky N (2002). Identification of PSE and OXA beta-lactamase genes in Pseudomonas aeruginosa using PCR restriction fragment length polymorphism. J. Antimicrob. Chemother.50:11-18.

Bonnet R (2004). Growing group of extended-spectrum betalactamases: the CTX-M enzymes. Antimicrob. Agents. Chemother. 48:1-14.

Carbonne A, Arnaud I, Maugat S, Marty N, Dumartin C, Bertrand
X, Bajolet O, Savey A, Fosse T, Eveillard M, Sénéchal H, Coignard $\mathrm{B}$, Astagneau P, Jarlier V;MDRB Surveillance National Steering Group (BMR-Raisin) (2013). National multidrug-resistant acteria (MDRB) surveillance in France through the RAISIN network: a 9 year experience. J. Antimicrob. Chemother. 68:954-59.

Celenza C, Pellegrini G, Caccamo M, Segatore B, Amicosante G, Perilli M (2006). Spread of bla(CTX-M-type) and bla(PER-2) betalactamase genes in clinical isolates from Bolivian hospitals. J. Antimicrob. Chemother.57:975-978.

Chanawong A, Lulitanond A, Kaewkes W, Lulitanond VL, Srigulbutr S, Homchampa P (2007).CTX-M extended-spectrum beta-lactamases among clinical isolates of Enterobacteriaceae in a Thai university hospital. Southeast. Asian. J. Trop. Med. Public. Health. 38:493500.

Chen X, Yuan M, Li GX, Chen Y, Yu HL, Li J (2013). Dissemination of int1 gene and ISCR1 and their relations with multi-drug resistance in clinical isolates. China J. Znoonoses. 29: 646-652.

Dhanji H, Doumith M, Hope R, Livermore DM, Woodford N (2011). ISEcp1-mediated transposition of linked blaCTX-M-3 and blaTEM-1b from the Incl1 plasmid pEK204 found in clinical isolates of Escherichia coli from Belfast, UK. J. Antimicrob. Chemother. 66:2263-5.

Drees M, Pineles L, Harris AD, Morgan DJ (2014). Variation in definitions and isolation procedures for multidrug-resistant Gramnegative bacteria: a survey of the society for healthcare epidemiology of America research network. Infect Control Hosp. Epidemiol. 35:3626.

Han H, Zhou HJ, Li HS, Gao Y, Lu Zhi, Hu KX, Xu BL (2013). Optimization of pulse-field gel electrophoresis for subtyping of Klebsiella pneumoniae. Int. J. Environ. Res. Public Health 10:272031

Johnson JR, Johnston B, Clabots C, Kuskowski M, Castanheira M (2010). Escherichia coli sequence type ST131 as the major cause of serious multidrug-resistant E. coli infections in the United States. Clin. Infect. Dis. 51:286-94.

Kiiru J, Butaye P, Goddeeris BM, Kariuki S (2013). Analysis for prevalence and physical linkages amongst integrons, ISEcp1, ISCR1, Tn21 and Tn7 encountered in Escherichia colistrains from hospitalized and non-hospitalized patients in Kenya during a 19-year period (1992-2011). BMC. Microbiol.13:109.

Kiratisin P, Apisarnthanarak A, Saifon P, Laesripa C, Kitphati R, Mundy LM (2007). The emergence of a novel ceftazidime-resistant CTX-M extended-spectrum beta-lactamase, CTX-M-55, in both communityonset and hospital-acquired infections in Thailand. Diagn. Microbiol. Infect. Dis. 58:349-55.

Kumarasamy KK, Toleman MA, Walsh TR, Bagaria J, Butt F, Balakrishnan R, Chaudhary U, Doumith M, Giske CG, Irfan S, Krishnan P, Kumar AV, Maharjan S, Mushtaq S, Noorie T, Paterson DL, Pearson A, Perry C, Pike R, Rao B, Ray U, Sarma JB, Sharma M, Sheridan E, Thirunarayan MA, Turton J, Upadhyay S, Warner M, Welfare W, Livermore DM, Woodford N (2010). Emergence of a new antibiotic resistance mechanism in India, Pakistan, and the UK: a molecular, biological, and epidemiological study. Lancet. Infect. Dis. 10:597-602.

Li B, Yi Y, Wang Q, Woo PCY, Tan L, Jing H, Gao GF, Liu CH (2012). Analysis of drug resistance determinants in Klebsiella pneumoniae isolates from a tertiary-care hospital in Beijing,China. Plos.ONE. 7: e42280.

Magiorakos AP, Srinivasan A, Carey RB, Carmeli Y, Falagas ME, Giske CG, Harbarth S, Hindler JF, Kahlmeter G, Olsson-Liljequist B, Paterson DL, Rice LB,Stelling J, Struelens MJ, Vatopoulos A, Weber JT, Monnet DL (2012). Multidrug-resistant, extensively drug-resistant and pandrug-resistant bacteria: an international expert proposal for interim standard definitions for acquired resistance. Clin. Microbiol. Infect. 18: 268-81.

Pai H, Lyu S, Lee JH, Kim J, Kwon Y, Kim JW, Choe KW (1999). Survey of extended-spectrum beta-lactamases in clinical isolates of Escherichia coli and Klebsiella pneumoniae: prevalence of TEM-52 in Korea. J. Clin. Microbiol. 37:1758-63.

Pastera'n F, Rapoport M, Petroni A, Faccone D, Corso A, Galas $M$, Vázquez M, Procopio A, Tokumoto M, Cagnoni V (2006). Emergence of PER-2 and VEB-1a in Acinetobacter baumannii strains 
in the Americas. Antimicrob. Agents. Chemother. 50:3222-24.

Parveen MR, Manivannan S, Harish BN, Parija SC (2012). Study of CTX-M type of extended spectrum b-lactamase among nosocomial isolates of Escherichia coli and Klebsiella pneumoniae in south India. Indian J. Microbiol. 52:35-40.

Paterson DL, Hujer KM, Hujer AM, Yeiser N, Bonomo MV, Rice LB, Bonomo RA; International Klebsiella Study Group (2003). International Klebsiella Study Group: Extended-spectrum-lactamases in Klebsiella pneumoniae bloodstream isolates from seven countries: dominance and widespread prevalence of SHV-and CTX-M-TypeLactamases. Antimicrob. Agents Chemother. 47:3554-60.

Peirano G, Pitout JD (2010).Molecular epidemiology of Escherichia coli producing CTX-M $\beta$-Lactamase:the worldwide emergence of clone ST131 O25:H4. Int. Antimicrob. Agents. 35:316-21.

Rossolini GM, Andrea DMM, Mugnaioli C (2008). The spread of CTXMtype extended-spectrum beta-lactamases. Clin. Microbiol. Infect.14 (Suppl1):33-41.

Sidjabat HE, Paterson DL, Adams-Haduch JM, Ewan L, Pasculle AW, Muto CA, Tian GB, Doi Y (2009).Molecular epidemiology of CTX-Mproducing Escherichia coli isolates at a tertiary medical center in western Pennsylvania. Antimicrob. Agents. Chemother. 53:4733-9.

Sievert DM, Ricks P, Edwards JR, Schneider A, Patel J, Srinivasan A, Kallen A, Limbago B, FridkinS, National Healthcare Safety Network (NHSN) Team andParticipating N HSN Facilities (2013). Antimicrobial-resistant pathogens associated with healthcare-associated infections: summary of data reported to the National Healthcare Safety Network at the Centers for Disease Control and Prevention, 2009-2010. Infect. Control. Hosp. Epidemiol. 34:1-14.

Smith SP, Manges AR, Riley LW (2008). Temporal changes in the prevalence of community-acquired antimicrobial-resistant urinary tract infection affected by Escherichia coli clonal group composition. Clin. Infect. Dis. 46:689-95.

Sturenburg E, Kuhn A, Mack D, Laufs R (2004). A novel extended spectrum beta-lactamase CTX-M-23 with a P167T substitution in the active site omega loop associated with ceftazidime resistance. J. Antimicrob. Chemother. 54:406-409. Tacconelli E, Cataldo MA, Dancer SJ, De Angelis G, Falcone M, Frank U, Kahlmeter G, Pan A, Petrosillo N, Rodríguez-Baño J, Singh N, Venditti M, Yokoe DS, Cookson B; European Society of Clinical Microbiology (2014). ESCMID guidelines for the management of the infection control measures to reduce transmission of multidrug-resistant Gramnegative bacteria in hospitalized patients. Clin. Microbiol. Infect. 20:155.

Tan HL, Wang Y, Cheng XQ, Huang YM, Zhang LJ (2014). Molecular epidemiological analysis of multi-drug resistant E.coli and K.pneumoniae isolates in Yunnan, China. Chin.Trop.Med.14:77781.(In Chinese)

Tian SF, Chu YZ, Chen BY, Nian H, Shang H (2011). ISEcp1 element in association with bla(CTX-M) genes of $E$. coli that produce extendedspectrum $\beta$-lactamase among the elderly in community settings. Enferm. Infecc. Microbiol. Clin. 29:731-4.
Vourli S, Giakkoupi P, Miriagou V, Tzelepi E, Vatopoulos AC, Tzouvelekis LS (2004). Novel GES/IBC extended-spectrum betalactamase variants with carbapenemase activity in clinical enterobacteria. FEMS. Microbiol. Lett. 234:209-213

Wang GQ, Huang T, Surendraiah KPM, Wang K, Komal R, Zhuge J, Chern CR, Kryszuk AA, King C, Wormser GP (2013). CTX-M $\beta$ Lactamase-producing Klebsiella pneumoniae in Suburban New York, New York, USA. Emerg. Infect. Dis.19:1803-10.

Wang XR, Chen JC, Kang Y, Jiang N, An SC, Gao ZC (2012). Prevalence and haracterization of plasmid-mediated blaESBL with their genetic environment in Escherichia coli and Klebsiella pneumoniae in patients with pneumonia. Chin. Med. J.125: 894-900.

Wei ZQ, Chen YG, Yu YS, Lu WX, Li LJ (2005). Nosocomial spread of multi-resistant Klebsiella pneumoniae containing a plasmid encoding multiple beta-lactamases. J. Med. Microbiol. 54:885-8.

Weisburg WG, Barns SM, Peleetier DA, Lane DJ (1991). 16S ribosomal DNA amplification for phylogenetic study. J. Bacteriol.173:697-703.

Yan JJ, Tsai SH, Chuang CL, Wu JJ (2006). OXA-type betalactamases among tended-spectrum cephalosporin-resistant Pseudomonas aeruginosa isolates in a university hospital in southern Taiwan. J. Microbiol. Immunol. Infect. 9:130-134.

Zhou TY, Ni YX (2010). "Protocol of bacterial isolation of clinical specimen: Manual of Clinical Microbiology Laboratory”. pp. 325-412. (In Chinese). 